persiguiendo un mayor beneficio. La citorreducción con escleroterapia percutánea, radiofrecuencia y embolización hepática son tratamientos alternativos ${ }^{2,7}$. Recientemente, el uso de análogos de la somatostatina con estabilización del tumor en al menos 2/3 de los pacientes ha surgido como la terapia alternativa de la enfermedad avanzada ${ }^{6,8}$

\section{B I B L I O G R A F Í A}

1. Raslan WF, Ro JY, Ordonez NG, Amin MB, Troncoso P, Sella A, et al. Primary carcinoid of the kidney. Immunohistochemical and ultrastructural studies of five patients. Cancer. 1993;72: 2660-6.

2. Krishnan B, Truong LD, Saleh G, Sirbasku DM, Slavin KM. Horseshoe kidney is associated with an increased relative risk of primary renal carcinoid tumor. J Urol. 1997;157:2059-66.

3. Gross MI, Berger I. Primary metastatic renal carcinoid in a young male patient: A contribution to the differential diagnosis of neuroendocrine tumors. Pathologue. 2007;28:285-90.

4. Romero FR, Rais-Baharami S, Permpongkosol S, Fine SW, Kohanin S, Jarrett TW. Primary carcinoid tumors of the kidney. J Urol. 2006. 176-2359-66.
5. Quinchon JF, Aubert S, Biserte J, Lemaitre L, Gosselin B, Leroy X. Primary atypical carcinoid of the kidney: A classification is needed. Pathology. 2003;35:353-5.

6. Motta L, Candiano G, Pepe P, Panella P, Galia A, Aragona A. Neuroendocrine tumor in a horseshoe kidney. Case report and updated follow-up of cases reported in the literature. Urol Int. 2004;73:361-4.

7. McCaffrey JA, Reuter V, Herr HW, Macapinlac HA. Carcinoid tumor of the kidney: The use of somatostatin receptor scintigraphy in diagnosis and management. Urol Oncol. 2000;5:108-11.

8. Arnold R, Simon B, Wied M. Treatment of neuroendocrine GEP tumours with somatostatin analogues. Digestion. 2000;62: 84-91.

J. Cabral Ribeiro*, L. Sousa y A. Ribeiro Santos

Department of Urology, Hospital de São Marcos, Braga, Portugal

*Autor para correspondencia.

Correo electrónico: jcabral@hsmbraga.min-saude.pt

(J. Cabral Ribeiro).

\title{
Empiema del muñón ureteral. A propósito de un caso y revisión de la literatura
}

\section{Ureteral stump empyema. A report of a case and review of the literature}

\section{Sr. Director:}

Es infrecuente que el muñón ureteral remanente tras cirugía renal presente complicaciones tardías infecciosas en ausencia patología ureteral previa.

Se presenta el caso clínico de una mujer de 43 años con antecedentes de apendicectomía y de nefrectomía laparoscópica derecha realizada 8 años antes por atrofia hidropionefrótica asociada a litiasis e infecciones recurrentes por Escherichia Coli secundarias a estenosis de la unión pieloureteral, pero sin la evidencia de reflujo vesicoureteral.

Tras más de 7 años sin síntomas urológicos presentó cistitis de repetición (4 episodios en 3 meses en los cuales se realizaron 3 urocultivos negativos y una ecografía urológica sin alteraciones) a pesar del tratamiento con fosfomicina trometamol, norfloxacino y cefuroxima por lo que requirió tratamiento supresor nocturno durante 6 meses con nitrofurantoína ( $50 \mathrm{mgr} / 24 \mathrm{~h}$ ) durante los cuales estuvo asintomática.

Tres semanas después de finalizar este tratamiento acudió a urgencias con cuadro febril e importante afectación del estado general. En la exploración física presentaba dolor en ambas fosas ilíacas y en fosa renal izquierda. Se realizaron las siguientes pruebas de laboratorio: una analítica sanguínea con una hemoglobina de $12 \mathrm{~g} / \mathrm{dl}$, leucocitos $13.800 / \mathrm{mm}^{3}$ con $85,9 \%$ de neutrófilos y creatinina de $0,9 \mathrm{mg} / \mathrm{dl}$; y un sedimento de orina que no presentó elementos patológicos. El urocultivo realizado fue negativo. En la radiografía de abdomen se identificaban grapas de nefrectomía en fosa renal derecha y un DIU. Con estos hallazgos se realizó una ecografía abdominal objetivándose una estructura tubular hipoecoica de aproximadamente $2,5 \mathrm{~cm}$ de diámetro en FID, de localización retroperitoneal adyacente a vasos ilíacos derechos y lateral a útero y vejiga, que con el estudio doppler no presentó flujo. Con esta prueba se descartó una uropatía obstructiva en el riñón izquierdo y la presencia de litiasis urinarias.

Con la sospecha de colección se realizó una TC multicorte 64 coronas con contraste oral e intravenoso en fase venosa donde se observó una estructura tubular que comenzaba a nivel de los clips quirúrgicos de la nefrectomía derecha, descendía por el retroperitoneo lateralmente a la vena cava abdominal y cruzaba anteriormente la vena iliaca común derecha siguiendo el trayecto del uréter (fig. 1A). Esta estructura presentaba un 


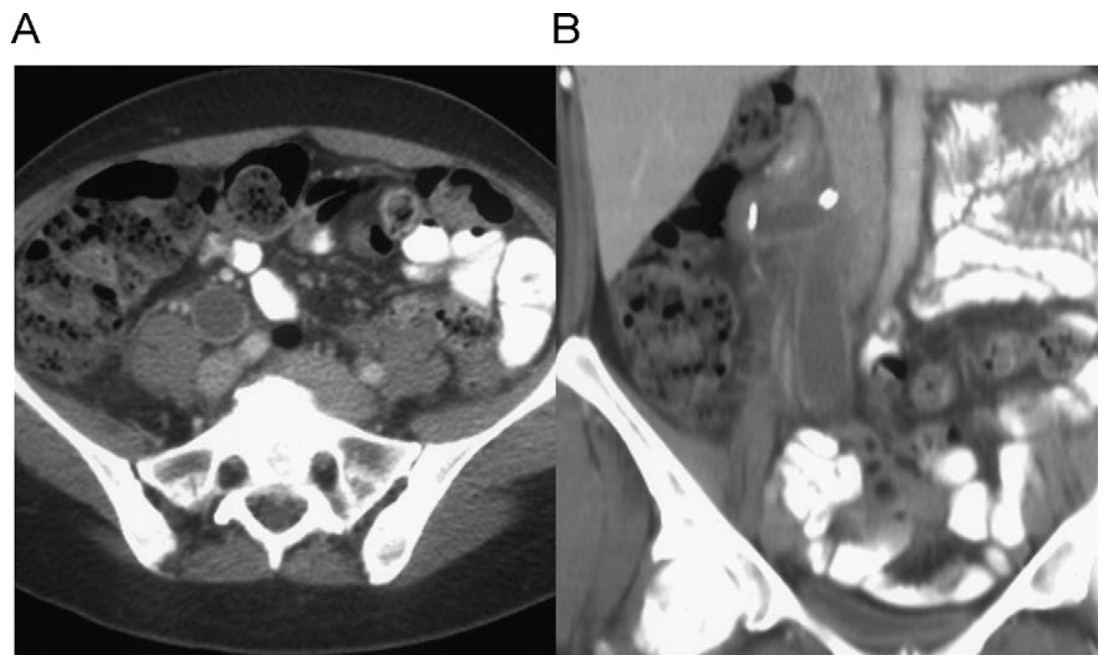

Figura 1 - A) TC corte axial donde se visualiza una estructura redondeada de $2 \mathrm{~cm}$ de diámetro, anteromedial al músculo psoas y adyacente a los vasos ilíacos. B) TC reconstrucción coronal donde se observa dilatación del muñón ureteral con realce de sus paredes y contenido hipodenso, que se extiende distalmente desde los clips quirúrgicos.

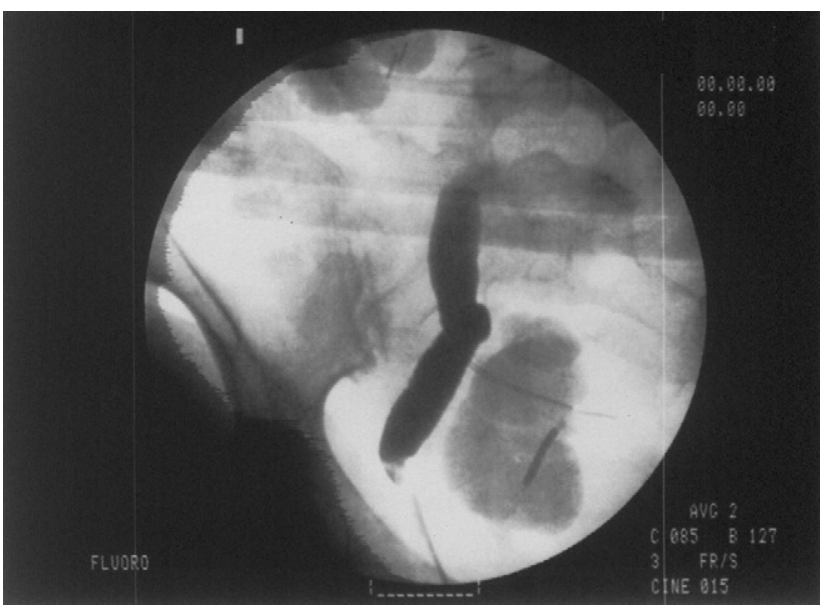

Figura 2 - Ureterografía retrógrada derecha donde se observa una estenosis ureteral del tramo submural seguida de una dilatación proximal. Se visualiza contraste en las asas intestinales de la TC previa.

realce de la pared así como contenido hipodenso, con un diámetro de $2 \mathrm{~cm}$ (fig. 1B), la cual se correspondía con el muñón ureteral con signos inflamatorios infecciosos.

A continuación se realizó una cistoscopia, objetivándose un meato ureteral derecho puntiforme y una ureterografía retrógrada derecha en la cual se observaba una estenosis del tramo submural seguida de dilatación completa del resto del uréter hasta los clips quirúrgicos (fig. 2). Se colocó un catéter ureteral doble J drenándose gran cantidad de pus. El catéter se retiró mes y medio después sin incidencias y se indicó una ureterectomía laparoscópica.

El empiema del muñón ureteral o hidropiouréter es una enfermedad poco común (incidencia del $0,8 \%{ }^{1}$ ). El intervalo de tiempo entre la intervención quirúrgica y la aparición de los síntomas puede variar entre meses y años, en nuestro caso la paciente había sido intervenida 8 años antes. La patología que puede presentar el muñón ureteral es diversa. Algunos autores describen el síndrome del muñón ureteral ${ }^{1}$, en el que incluyen el empiema, la ureteritis crónica o el tumor de células transicionales ${ }^{2,3}$. El muñón ureteral no suele presentar patología si en el momento de la cirugía no presentaba alteraciones. Sin embargo uréteres crónicamente infectados, obstruidos, asociados a litiasis o a reflujo distal pueden producir sintomatología meses o años después ${ }^{1,4}$.

Tradicionalmente, la enfermedad ureteral ha sido estudiada mediante urografía intravenosa o ureteropielografía retrógrada, mientras que en la actualidad han sido reemplazadas por otras técnicas (ecografía, TC y RM). En el estudio de Pollack et $\mathrm{al}^{2}$, publicado en 1982, se diagnosticó incorrectamente a la mayor parte de los pacientes. Muchos de ellos se describen como hallazgo quirúrgico. Hoy en día su diagnóstico es más precoz aunque inicialmente esta enfermedad no sea sospechada, dado que sus síntomas suelen ser atribuidos a enfermedades no urológicas. De ahí la importancia de incluirlo en los diagnósticos diferenciales en los pacientes con este antecedente quirúrgico, para evitar una demora diagnóstica. Esta afección cursa con dolor abdominal bajo e infecciones del tracto urinario ${ }^{2}$, siendo los hallazgos de la exploración física y analíticos inespecíficos.

El TC multicorte con contraste i.v. es la mejor técnica de imagen para el diagnóstico del empiema ureteral. La reconstrucción coronal es la más adecuada para el estudio de esta patología, ya que nos permite visualizar el trayecto en su totalidad. Además de demostrar la existencia de un empiema ureteral (dilatación elíptica del muñón ureteral, con realce y engrosamiento de la pared ${ }^{5}$ ) nos permite descartar litiasis, compresiones extrínsecas de la pared o tumores en el muñón.

La ecografía nos puede orientar hacia su diagnóstico, pero dado que el uréter sigue un trayecto retroperitoneal profundo, no nos permite visualizarlo en su totalidad. Sí puede ser útil para descartar otro tipo de patología que puede causar una sintomatología similar, como patología ginecológica, digestiva o urinaria. En nuestro caso, era importante considerar la posibilidad de patología ginecológica como la enfermedad 
inflamatoria pélvica, dada la edad de la paciente y que presentaba factores de riesgo como la utilización de DIU. También se puede realizar una RM potenciada en T2 sin contraste $^{6}$ o una RM urográfica tras la administración de contraste i.v. en la fase excretora.

En un estudio Kim $\mathrm{YJ}^{7}$ concluye que es necesario el seguimiento a largo plazo del uréter remanente, ya que en 318 pacientes nefrectomizados por patología benigna diagnosticó 8 tumores, 6 de ellos con antecedentes de pionefrosis y 2 de tuberculosis renal (resultado anatomopatológico de 6 carcinomas de células transicionales y 2 escamosos) y con un periodo medio libre de enfermedad de 76,5 meses. En 1994 Fariña L.A. aportó 2 casos de aparición de neoplasia en el muñón ureteral, uno de ellos 38 años después de una nefrectomía por tuberculosis renal ${ }^{8}$.

El tratamiento definitivo de esta patología es la escisión del uréter remanente. En nuestro caso se realizó en un primer tiempo tratamiento mediante drenaje de la colección, quedando pendiente la realización de la ureterectomía.

B I B L I O G R A F Í A

1. Ikeda D, Matsutani R, Fukuda M, Fuse H, Hirano S. Transurethral fulguration for empyema of ectopic ureteral stump. Int J Urol. 2003;10:664-6.

2. Pollack HM, Banner MP, Popky GL. Radiologic evaluation of the ureteral stump. Radiology. 1982;144:225-30.
3. Prajsner A, Szewczyk W, Gawron R. Empyema of retained ureteral stump with gross stone 20 years after nephrectomy. Urology. 2009;73:993-4.

4. Barroso Jr U, Calado AA, Filho MZ. The role of refluxing distal ureteral stumps after nephrectomy. J Pediatr Surg. 2002;37:653-6.

5. Persad R, Kamineni S, Mouriquand PD. Recurrent symptoms of urinary tract infection in eight patients with refluxing ureteric stumps. Br J Urol. 1994;74:720-2.

6. Leyendecker JR, Barnes CE, Zagoria RJ. MR urography: techniques and clinical applications. Radiographics. 2008;28:23-46. discussion 46-7.

7. Kim YJ, Jeon SH, Huh JS, Chang SG. Long-term follow-up of ureteral stump tumors after nephrectomy for benign renal disease. Eur Urol. 2004;46:748-52.

8. Fariña LA, Caparros Sariol J, Villavicencio Mavrich H. Carcinoma of the residual ureter many years after nephrectomy: an evasive cause of hematuria and a high risk tumor. Arch Esp Urol. 1994;47:581-4.

L. Labairu-Huerta ${ }^{a}, *$ A. Burguete-Moriones ${ }^{b}$,

J. Zabalza-Unzuéb y V. Grasa-Lanau ${ }^{\mathrm{a}}$

${ }^{a}$ Servicios de Urología, Hospital de Navarra, Pamplona, España

${ }^{\mathrm{b}}$ Servicios de Radiodiagnóstico, Hospital de Navarra, Pamplona, España

*Autor para correspondencia.

Correo electrónico: luislabairu@hotmail.com

(L. Labairu-Huerta).

\section{Metástasis en la vagina como primer síntoma de un carcinoma de células renales}

\section{Vaginal metastasis as the first sign of renal cell carcinoma}

\section{Sr. Director:}

El carcinoma de células renales (CCR) tiene frecuentemente un curso clínico impredecible, y aunque son frecuentes las metástasis a distancia en el momento del diagnóstico, incluso como forma de presentación del tumor, son excepcionales en la vagina y hay menos de 100 casos descritos, según la literatura médica revisada ${ }^{1-10}$.

Presentamos un caso correspondiente a una mujer de 53 años, sin antecedentes de interés, que consultó por sangrado vaginal. En la exploración ginecológica se observó una tumoración polipoide, necrosada, de aspecto infiltrativo en la cara anterior y en el fondo del saco derecho de la vagina que se biopsió. Histológicamente, fue diagnosticado de carcinoma de células claras (CCC) muy sugestivo de origen renal (queratina, vimentina, EMA, CD10 y anticuerpo para CCR positivo) (fig. 1), por lo que se recomendó valorar clínica y radiológicamente esta posibilidad. La tomografía computarizada abdominopelviana demostró un tumor renal de $12 \mathrm{~cm}$ de diámetro máximo que ocupaba el tercio medio y superior del riñón derecho, invadía el seno renal (fig. 2) y mostraba importante circulación colateral, sobre todo venosa (fig. 2, flecha), que se dirigía a través de la vena ovárica derecha hacia la pelvis (fig. 2, *). No se observó patología a ningún otro nivel y la gammagrafía ósea fue negativa. No se realizó nefrectomía por la rápida progresión de la metástasis vaginal y la paciente recibió radioterapia pelviana con finalidad hemostática. A los 10 meses del diagnóstico inicial y después de recibir tratamiento con radioterapia, la paciente presentó progresión de la enfermedad con aumento de 